\title{
Optimal management of a biochemical incomplete response to therapy in differentiated thyroid cancer: aggressive treatment or cautious observation?
}

\author{
R. Michael Tuttle
}

Received: 24 December 2013/Accepted: 13 February 2014/Published online: 11 March 2014

(C) Springer Science+Business Media New York 2014

The last 10 years has seen a renewed interest in a risk adapted management approach to differentiated thyroid cancer. We now view risk stratification as an ongoing, dynamic process that begins with initial estimates of the risk of death from thyroid cancer and risk of recurrent/ persistent disease for each patient [1]. These initial risk estimates are used to guide early management decisions and plan follow-up studies that allow us to define the response to initial therapy for each patient. As information is accumulated during follow-up, we actively modify the initial risk estimates based on the clinical course of the disease and the patient's individual response to therapy. To facilitate classification and communication, we have previously proposed a nomenclature that can be used to describe response to therapy as either [1-3]

- Excellent response: no clinical, biochemical, or structural evidence of disease,

- Biochemical incomplete response: abnormal thyroglobulin $(\mathrm{Tg})$ or rising anti-thyroglobulin antibody levels in the absence of localizable disease,

- Structural incomplete response: persistent or newly identified locoregional or distant metastases, or

- Indeterminate response: non-specific biochemical or structural findings which cannot be confidently classified as either benign or malignant.

These response to therapy categories have important clinical implications [2-4]. An excellent response to therapy is associated with a subsequent risk of recurrence of 1-2\%. Serum $\mathrm{Tg}$ values often spontaneously fall in the

R. M. Tuttle ( $₫)$

Endocrinology Service, Memorial Sloan-Kettering Cancer

Center, New York, NY 10021, USA

e-mail: tuttlem@mskcc.org biochemical incomplete response category with only $10-15 \%$ developing structural evidence of disease. Unfortunately, even with additional treatments, the structural incomplete response to therapy cohort usually remains with persistent biochemical or structural evidence of disease. The indeterminate response cohort is more heterogeneous with about $15-20 \%$ of the patients in this cohort being reclassified as having biochemical or structural evidence of disease during follow-up $[2,5]$.

In order to better define the clinical significance of low level TSH-stimulated $\mathrm{Tg}$ values that are identified without a structural disease correlate (corresponding to either the indeterminate or biochemical incomplete response described above), Pitoia et al. [6] report the outcomes after a median follow-up period of 6 years in a retrospective cohort of 32 well-characterized differentiated thyroid cancer patients demonstrating a TSH-stimulated thyroglobulin value $>1 \mathrm{ng} / \mathrm{mL}$ despite having an undetectable $\mathrm{Tg}$ on levothyroxine suppression $(<1 \mathrm{ng} / \mathrm{mL})$ and no structural/functional evidence of disease. Importantly, they analyzed the outcomes a based on the TSH-stimulated $\mathrm{Tg}$ values (Group 1 -stimulated $\mathrm{Tg} 1-2 \mathrm{ng} / \mathrm{mL}$ vs. Group 2 -stimulated $\mathrm{Tg} 2-10 \mathrm{ng} / \mathrm{mL}$ vs. Group 3-stimulated $\mathrm{Tg}>10 \mathrm{ng} / \mathrm{mL}$ ). At final follow-up, all of the patients in Group 1 were classified as no evidence of disease, 4/17 (24\%) of patients in Group 2 developed metastatic cervical lymph nodes $1-3.5$ years after initial assessment, and all patients in Group 3 had persistent evidence of biochemical $(89 \%)$ or structural disease $(11 \%$, one patient developed a metastatic cervical lymph node 3 years after initial therapy). As commonly happens, persistent biochemical evidence disease persists despite surgical removal of the lymph node metastases in all five patients that developed structural evidence of disease during follow-up. These data are consistent with our prior studies that 
demonstrate that only a minority of patients with low level abnormal $\mathrm{Tg}$ values will subsequently develop clinically apparent structural disease. And unfortunately, even when they are subjected to additional treatments, the most common outcome is not a biochemical cure, but rather ongoing, persistent low level Tg values.

These observations must make us re-evaluate our current aggressive management approach to the detection and treatment of minimal residual disease. We must discard the traditional belief that untreated occult disease is invariably a progressive condition that always results in harm to the patient. For years, many of us firmly believed that "even modest increments in detecting occult tumor that is amenable to treatment will benefit many patients." [7]. However, it now appears that only a minority of patients with a biochemical incomplete response to therapy develops clinically significant disease. Additionally, we now appreciate that well-meaning additional therapies often do not produce a cure providing evidence that this occult disease is not as "amenable to treatment" as we had hoped. As a result, I no longer believe that aggressive treatment of occult disease identified by highly sensitive detection methods is benefitting the majority of patients. Nonetheless, I have no doubt that minimal disease detection does significantly improve outcomes in some patients.

Over the years, my clinical management of low level persistent $\mathrm{Tg}$ (TSH-stimulated $\mathrm{Tg}<10 \mathrm{ng} / \mathrm{mL}$, and/or suppressed Tg $0.2-0.9 \mathrm{ng} / \mathrm{mL}$ ) identified in the absence of structural or functional evidence of disease has evolved from aggressive imaging and early re-treatment to a more cautious observation approach.

1. TSH is maintained in the $0.5-1.0 \mathrm{mIU} / \mathrm{mL}$ range in patients initially classified as ATA low or intermediate risk with slightly more suppression (TSH $0.1-0.5 \mathrm{mIU} / \mathrm{mL}$ ) in the ATA high risk patients or in patients that may be poor $\mathrm{Tg}$ producers (poorly differentiated thyroid cancer).

2. Neck US is obtained at $3-5$ year intervals provided the non-stimulated Tg remains $<1 \mathrm{ng} / \mathrm{mL}$ in the absence of anti-Tg antibodies.

3. Additional structural or functional imaging is considered for the few patients that have a rising Tg over time or development of anti-Tg antibodies during followup.

4. Diagnostic RAI scanning is considered in patients with consistently rising TG values or with newly detected $\mathrm{Tg}$ antibodies in an effort to identify RAI avid metastatic disease.

5. Empiric RAI therapy in the setting of a negative diagnostic scan is rarely recommended except when TG values are dramatically rising without a structural correlate.
6. Repeat stimulated Tg testing is considered at 3-5 years intervals (or measurement of TG on a highly sensitive $\mathrm{Tg}$ assay that reliably reads $<0.2 \mathrm{ng} / \mathrm{mL}$ ) in order to identify those patients who can be reclassified as having an excellent response.

This more cautious approach avoids the side effects of aggressive TSH suppression, the risks associated with excess imaging, and ineffective additional treatments, while at the same time providing a monitoring program designed to identify the few patients destined to develop clinically significant disease progression that may warrant additional imaging or treatment. The time has come for us to think about differentiated thyroid cancer as a chronic disease in which our highly sensitive detection tools often identifies occult disease that harbors only a very low potential to cause substantial harm. Additional studies are needed to more precisely define which patients with differentiated thyroid cancer are most likely to benefit from minimal disease detection and treatment.

Conflict of interest Consultant to Genzyme/Sanofi

\section{References}

1. H. Tala, R.M. Tuttle, Contemporary post surgical management of differentiated thyroid carcinoma. Clin. Oncol. (R Coll Radiol) 22, 419-429 (2010)

2. R.M. Tuttle, H. Tala, J. Shah, R. Leboeuf, R. Ghossein, M. Gonen, M. Brokhin, G. Omry, J.A. Fagin, A. Shaha, Estimating risk of recurrence in differentiated thyroid cancer after total thyroidectomy and radioactive iodine remnant ablation: using response to therapy variables to modify the initial risk estimates predicted by the new American thyroid association staging system. Thyroid 20, 1341-1349 (2010)

3. F. Vaisman, H. Tala, R. Grewal, R.M. Tuttle, In differentiated thyroid cancer, an incomplete structural response to therapy is associated with significantly worse clinical outcomes than only an incomplete thyroglobulin response. Thyroid 21, 1317-1322 (2011)

4. M.G. Castagna, F. Maino, C. Cipri, V. Belardini, A. Theodoropoulou, G. Cevenini, F. Pacini, Delayed risk stratification, to include the response to initial treatment (surgery and radioiodine ablation), has better outcome predictivity in differentiated thyroid cancer patients. Eur. J. Endocrinol. 165, 441-446 (2011)

5. F. Vaisman, D. Momesso, D. Bulzico, C. Pessoa, F. Dias, R. Corbo, M. Vaisman, R. Tuttle, Spontaneous remission in thyroid cancer patients after biochemical incomplete response to initial therapy. Clin. Endocrinol. (Oxf) 77, 132-138 (2012)

6. Pitoia F, Erika A, Tala Jury HP, Fernanda B, Carolina U, Graciela C 2014 Biochemical persistence in thyroid cancer: is there anything to worry about? Endocrine (in press)

7. E.L. Mazzaferri, R.J. Robbins, C.A. Spencer, L.E. Braverman, F. Pacini, L. Wartofsky, B.R. Haugen, S.I. Sherman, D.S. Cooper, G.D. Braunstein, S. Lee, T.F. Davies, B.M. Arafah, P.W. Ladenson, A. Pinchera, A consensus report of the role of serum thyroglobulin as a monitoring method for low-risk patients with papillary thyroid carcinoma. J. Clin. Endocrinol. Metab. 88, 1433-1441 (2003) 